\section{Corneal dystrophies and degenerations: a molecular genetics approach}

Edited by Ming X Wang. 151 pp. plus 1 CD. Oxford University Press in cooperation with the American Academy of Ophthalmology, 2003.

European Journal of Human Genetics (2004) 12, 1083. doi:10.1038/sj.ejhg.5201259

The largest contribution to the refractive power of the eye does not come from the lens, but from the cornea. Opacity of this part of the eye is one of the most important causes of visual impairment. The book edited by Dr Wang is a largely successful attempt to give a summary of a group of inherited diseases affecting the cornea. The aim of the volume is to educate the reader about all aspects of these diseases from the elucidation of the molecular pathogenesis to the current therapeutical options. In the preface, it is explicitly stated that the practicing ophthalmologist is addressed by the book. Does it succeed in informing 'the' ophthalmologist about genetic issues, and is it also of interest for 'the' geneticist? The material is organized in six chapters: The first chapter gives a summary of the genes known to be mutated in several inherited corneal dystrophies. The chapter focuses on the gene encoding keratoepithelin (TGFBI, BIGH3), in which mutations have been identified in at least six clinically distinct inherited corneal dystrophies, and it also contains information about dystrophies not linked to the TGFBI gene on chromosome 5q. Very useful in this chapter are the tables listing the corneal dystrophies and their mode of inheritance, the mutations in the TGFBI gene (with information on genotype-phenotype correlation), and the corneal dystrophies not linked to the TGFBI gene. Chapters 2-4 review the corneal dystrophies of the corneal epithelium and Bowman's layer, corneal stroma, and corneal endothelium, respectively. The organization of the material about the diseases is somewhat different between the different chapters, but by and large there is a paragraph on the clinical picture, on the histopathology, on genetics and disease mechanisms, and on therapeutical options and patient management for the described corneal dystrophies. Chapter 5 gives an overview about corneal and conjunctival degenerations, most of which are not inherited, but have an exogenous cause. Chapter 6 summarizes the use of excimer laser surgery in treating corneal dystrophies. The book also contains questions for self-study examination, and a detailed and very useful index. Also included is a CD with all figures of the book plus several additional figures.

The coverage of the literature is relatively complete up to the year 2000, and and a few publications of the contributing authors as recent as 2001 and 2002 are also included. Consequently, very recent developments in the field are missed; for instance, the identification by Heon et al (2002) of the VSX1 gene being mutated in posterior polymorphous dystrophy and some familial forms of keratoconus. In the more clinical chapters, the use of the genetic terminology is sometimes a bit unorthodox. This is, for instance, the case for congenital hereditary endothelial dystrophy, p 73: the gene is said to be identified, but only a chromosomal localization is known, and the gene is still completely unknown. Chapter 2 contains a short discussion of inherited syndromes in which corneal dystrophies are only one of several different manifestations. One of the conditions not mentioned in the book is Peters anomaly, which is covered in other reviews of the subject, for instance, by Vincent et al (2003) Dev Ophthalmol 37: 50-66. Of course, it is always a matter of discussion what should be included: Peters anomaly results from mutations in at least four different genes, which are all different from the genes identified for the other corneal dystrophies. It would also be a gain if information on the incidence of these relatively rare inherited conditions could be given.

The editor and many of the authors are optimistic that the identification of genes mutated in inherited corneal dystrophy will result in a more rational, biologic classification of these diseases. The fact that most of the clinical information is still organized following anatomical criteria, that different mutations in one gene can result in at least six clinically different diseases, and that at the moment mutations in at least 14 different genes can result in a corneal dystrophy, indicates that the identification of genetic causes of diseases can often also introduce a new level of complexity.

In conclusion, this book fits probably best into the ophthalmological library, as suggested by the editor. However, a human geneticist interested in corneal dystrophies, because he/she is involved in counselling or molecular analysis of a family with one of the conditions dealt with in this volume, will borrow the book so often from the ophthalmologist that it will almost certainly be necessary to acquire a copy for the genetic library, too.

M Hergersberg*

Kantonsspital Aarau, Zentrum für Labormedizin, Kantonsspital, Aarau, CH-5001, Switzerland

${ }^{*}$ Correspondence: Dr M Hergersberg, Kantonsspital Aarau, Zentrum für Labormedizin, Kantonsspital, Aarau, CH-5001, Switzerland. Tel: 004162838 5303; Fax: 0041628385399 ; E-mail: martin.hergersberg@ksa.ch 